\title{
A Brief Analysis of Japanese-Chinese Translation Studies of the Popular Science Text of Traditional Chinese Pharmacology in Japan
}

\author{
Yan Li \\ Youjiang Medical University for Nationalities, Baise, China \\ Email: susanliyan8@163.com
}

How to cite this paper: Li, Y. (2021). A Brief Analysis of Japanese-Chinese Translation Studies of the Popular Science Text of Traditional Chinese Pharmacology in Japan. Advances in Literary Study, 9, 21-25. https://doi.org/10.4236/als.2021.91004

Received: December 29, 2020

Accepted: January 17, 2021

Published: January 20, 2021

Copyright ( 2021 by author(s) and Scientific Research Publishing Inc. This work is licensed under the Creative Commons Attribution International License (CC BY 4.0).

http://creativecommons.org/licenses/by/4.0/

\section{(c) (i) Open Access}

\begin{abstract}
Chinese herbal medicine features strong national characteristics, and carries heavy cultural connotations. The use of Chinese herbal medicine originated in ancient China, which was called Japanese Kampo medicine after it was introduced into Japan. With the close cultural exchanges between China and Japan and the continuous improvement of medical technology, Chinese herbal medicine has become more and more appreciated and recognized by the Japanese for its unique charm. At the same time, the research on herbology in China and Japan has also been unprecedentedly developed. Based on the purpose of mutual learning and reference, this paper discusses the JapaneseChinese translation studies of the popular science text of traditional Chinese pharmacology in Japan.
\end{abstract}

\section{Keywords}

Translation Study, Popular Science Text, Japanese-Chinese Translation, Traditional Chinese Pharmacology, Chinese Herbal Medicine

\section{Introduction}

Currently, Guangxi is home to more than 4000 known medicinal plant species, accounting for about one-third of the national medicinal plant resources, of which Zhuang medicine and Yao medicine are the most distinctive. Due to the complex geographic environment and special climatic conditions, Baise, where the Zhuang ethnic group lives, has created a very rich resource of medicinal materials. Baise of Guangxi is known as the "natural traditional Chinese medicine library" (Wei, Nong, Mei, Zou, Shu, Li, Li, Wang, Liu, Su, Long, Huang, Ye, \& Peng, 2017). In 2019, the transaction volume of Chinese herbal medicines in the 
drug market among Dragon Boat Festival in Jingxi County, Baise, China reached more than 800 tons with on-site transaction volume of 6.8 million yuan. The vast majority of Japanese Kampo medicines come from China and Southeast Asian countries, and a few are self-produced or transplanted in Japan. Herbal medicine is mainly based on plant medicinal materials, a valuable asset accumulated by ordinary people in their long-term life experience. There are some differences between Chinese herbal medicine and Japanese Kampo medicine, such as those of homonym and synonym. In addition, because ordinary people have different experiences in their long-term lives, even though the drug name is the same, but the medicinal parts vary. The translation of herbal medicine not only promotes the dissemination of medical culture, but also broadens the horizons, promotes academic exchanges, and realizes medicinal value.

\section{Significance of Japanese-Chinese Translation of the Popular Science Text of Traditional Chinese Pharmacology in Japan}

The "Modernization of Traditional Chinese Medicine" strategy has been implemented for more than 20 years since it was proposed by the Ministry of Science and Technology in 1996. In the meantime, although traditional Chinese medicine has made some progress in terms of production technology and dosage form, the overall effect is barely satisfactory, and there are still numerous problems to be resolved, which hinder the further development of the traditional Chinese medicine industry in China. Japan, where traditional Chinese medicine was introduced, has achieved great success in the development of Japanese Kampo medicine in the past 40 years. There are about 200 Kampo medicine factories in Japan and more than 2000 Kampo preparations. 89\% of Japanese physicians prescribe Kampo medicines, and the prescriptions with Kampo medicine are increasing at a rate of $15 \%$ every year. At present, more than $80 \%$ of the 60,000 pharmacies in Japan engage in Kampo preparations, and Kampo medicines can basically be found in the prominent location in pharmacies. In the global trade volume of medicinal plants, Japan's share far exceeds that of China, occupying an absolute leading position. At present, Japanese Kampo medicines occupy 90\% of the world's sales of traditional Chinese medicine (Yang, Kamo, Nose, 2016). In view of this situation, we can learn from the development experience of Japanese medicinal plant cultivation. Therefore, the Japanese-Chinese translation of the Popular Science Text of traditional Chinese pharmacology in Japan is of great significance.

\section{Research on the Translation of the Popular Science Text of traditional Chinese Pharmacology in Japan is in the Ascendant}

At present, there are two outstanding problems in medical science translation in China. One is that translation practice is underestimated. Many people think that the style of popular science is simple and easy to understand, and transla- 
tion is relatively simple. The other is that there is a shortage of the research on popular science of medical science, especially when compared with the prosperity of translation of science and technology, the research on popular science of science translation is in an extremely "underdeveloped" stage (Lang, 2020). Translation only became a mature and independent subject in the 1990s. The foreign translation circle pays more attention to scientific translation, especially medical translation. For example, in 1998, the American Translators Association published the cornerstone of medical translation-Medical Translation Analects. Domestic research on medical translation is burgeoning, and medical translation studies mainly focus on the translation of medical vocabulary terms, and translation strategies and principles. However, the relevant research is mostly empirical theories, or uses the traditional translation theories such as those by $\mathrm{Yan} \mathrm{Fu}$ to make a superficial analysis, lacking grounded frontier theoretical guidance and correct strategic orientation.

The cultures of China and Japan run a long history, and the herbal medicine culture is even more extensive and profound. With the rapid development of information, translation has long been used as a bridge of communication and exchange. Therefore, the discussion on Japanese-Chinese medical translation will help promote cooperation and exchanges in this field. Medical translation serves as an important way to disseminate medical information. As medical science steps into the development of globalization, its demand is in growing numbers. Foreign translation scholars have come to realize the importance of the application of various translation theories to medical translation study. However, there are not many translation texts of traditional Chinese pharmacology in Japanese, there is a lack of text translation of traditional Chinese pharmacology from the perspective of translation theory, and the translation of popular science text in Japanese from the perspective of translation theory can merely be found.

\section{Guiding Theory in the Practice of Japanese-Chinese Translation of the Popular Science Text of Traditional Chinese Pharmacology in Japan}

Popular science texts are written by experts for laymen. Therefore, in order to clarify scientific principles, a variety of literary techniques must be utilized flexibly. Popular science writing, a genre that combines literature and science, uses popular and easy-to-understand language to spread and popularize scientific and technological knowledge to the public. The characteristics of popular science texts can be summarized as scientific, literary, popular and interesting. With the continuous deepening of global integration, popular science texts have become an important medium for people to acquire scientific knowledge from abroad. The famous American theorist and linguist Eugene Nida, known as the "father of translation theory", put forward a translation theory centering on "Functional Equivalence", exerting a profound influence on the Chinese and Western translation circles. Nida's Functional Equivalence Theory is the most representative of contemporary Western translation theories, and it is also the earliest contacted, 
most widely used and most influential theory in China (Zhang \& Yang, 2020).

As the core of Nida's translation theory, Functional Equivalence Theory has exerted a great impact on Chinese translation theory and translation practice. Nida emphasized that the response of target readers to the target text should be basically the same as that of original readers to the original text, which should first be equivalent in meaning, and then in style. In line with Nida's Functional Equivalence Theory, the translator's translation should be "readable and natural in wording, expressive in contents, and similar in reader's response". This requires the translator to meet the following three basic requirements: "It should convey the language and connotation information of the original text; the target language is fluent, natural, appropriate and easy to understand; the target language readers should react basically the same as the source language readers." Nida's Functional Equivalence Theory, as a receiver-oriented method, focuses on the function of translation, which had an important impact on many great translators (Jin, 2020).

In summary, the four characteristics of popular science texts determine the methods and strategies of popular science translation. First of all, the scientific and literary characteristics of popular science texts require translations featuring literary grace to be true to the original intention, and convey correct information. This coincides with the requirement of Functional Equivalence Theory to reproduce the original text information. Secondly, the popularity and interest of popular science texts require translators to pay attention to the feelings of target readers, and generate popular and natural target texts that conform to the target language norms. This is consistent with the value of Functional Equivalence Theory emphasizing reader's response. The Functional Equivalence Theory requires as much as possible to make recipients of the target text produce the same response as those of the original text. The popular science text of the traditional Chinese pharmacology is characterized with scientificity, literariness, popularity and interestingness. Therefore, in the translation practice, in order to ensure the equivalence of language functions, word-for-word translation is not required, and basic functional equivalence of vocabulary, sentences, etc., especially the translation of the names of Chinese medicine materials, should be guaranteed. Thus it can be seen that the Functional Equivalence Theory can provide effective guidance for the translation of the popular science text of traditional Chinese pharmacology. Nida's "Functional Equivalence" introduces a "closest natural equivalent" to reproduce the information and content of the original text, which, for translation of the popular science text of traditional Chinese pharmacology, is to translate using an objective, accurate, simple and natural language, enabling target readers to basically understand and appreciate the target text in a way that the original readers do (Cao, 2019).

\section{Conclusion}

Japan is a close neighbor of China, boasting relatively developed medical tech- 
nology. For a long time, China and Japan have had frequent exchanges in the field of Chinese herbology, which has been achieved through translation in most cases. However, due to the very professionalism of Chinese herbology, it has demanding requirements for the translator's own translation level and skills. Consequently, with increasingly frequent exchanges on Chinese herbology, actively exploring Japanese-Chinese medical translation techniques is of certain guiding and reference significance for promoting the in-depth cooperation and development of the two countries in this field. The Japanese-Chinese translation studies of the popular science text of traditional Chinese pharmacology in Japan provide a good channel for and serve as a bridge of the dissemination of cultures and information with regard to Chinese herbal medicine, laying a solid foundation for further development of herbal medicine.

\section{Fund Program}

The Project of Improving the Basic Scientific Research Ability of Young and Middle-aged Teachers in Colleges and Universities in Guangxi in 2019 (2019KY0563).

\section{Conflicts of Interest}

The author declares no conflicts of interest regarding the publication of this paper.

\section{References}

Cao, S. J. (2019). The Application of Functional Equivalence Theory in the English Translation of Treatise on Febrile Diseases. Hunan Journal of Traditional Chinese Medicine, 35, 82-84.

Jin, Y. Y. (2020). The Application of Eugene Nida's Functional Equivalence Theory in the English Version of Jin Yong's Novel. The Legend of the Condor Heroes. Data of Culture and Education, No. 14, 29-30.

Lang, Y. W. (2020). A Brief Analysis of the Translation Methods of "Non-Tech" Elements in Popular Science Texts Based on Nida's Functional Equivalence Theory. Sinogram Culture, No. 2, 141-142.

Wei, X., Nong, Z. F., Mei, X. P., Zou, M., Shu, J. L., Li, R. H., Li, C. J., Wang, M. H., Liu, J. X., Su, S. H., Long, W. Y., Huang, Z. C., Ye, Y. Y., \& Peng, Y. Y. (2017). Investigation and Thinking on Folk Medicine of Zhuang Medicine in Baise District, Guangxi. Journal of Medicine \& Pharmacy of Chinese Minorities, 23, 69-71.

Yang, J., Kamo, S., \& Nose, M. (2016). Development and Current Situation of Chinese Prescription Medicine in Japan. Chinese Traditional and Herbal Drugs, 47, 2771-2774.

Zhang, Y. F., \& Yang, Y. (2020). A Brief Analysis of the English Translation of Sichuan Dialects from the Perspective of Functional Equivalence Theory. English Square, No. 16, 30-33. 